\title{
Prevalência de tricomoníase e coinfecções em mulheres atendidas em dois centros de saúde em um município do Pará
}

\author{
Prevalence of trichomoniasis and co-infections in women treated at two health centers in a \\ municipality in Pará
}

\section{Prevalencia de tricomoniasis y coinfecciones en mujeres atendidas en dos centros de salud de un municipio de Pará}

Maiza Silva de Sousa ${ }^{1 *}$, Paulo Victor Gomes Pantoja ${ }^{1}$, Elisângela Silva Gomes ${ }^{1}$, Amanda Louise Rodrigues Oliveira ${ }^{1}$, Ana Oneide Brito Vasconcelos ${ }^{2}$, José Henrique Santos Silva ${ }^{3}$, Maria das Graças Carvalho Almeida ${ }^{1,4}$.

\section{RESUMO}

Objetivo: Determinar a prevalência de tricomoníase e coinfecções associadas em mulheres atendidas em dois centros de saúde localizados em um município do estado do Pará e correlacionar essas informações com variáveis sociodemográficas. Métodos: Trata-se de estudo retrospectivo, observacional, com abordagem quantitativa, realizado em duas unidades de saúde no estado do Pará, entre março e dezembro de 2019. A amostra foi constituída de 520 prontuários de mulheres atendidas entre janeiro de 2012 a dezembro de 2017, com idades entre 18 e 55 anos, apresentando diagnóstico para tricomoníase e/ou outras infecções. Resultados: A prevalência de T. vaginalis registrada para as duas unidades foi de $0,96 \%$. Quando associada a variáveis sociodemográficas, todas residiam no estado do Pará e se autodeclararam pardas; 0,58\% eram do lar, com idades entre $28-32$ anos, casadas e negavam o uso de anticoncepcional; apenas 0,38\% possuíam ensino médio completo. A prevalência de coinfecções envolvendo $T$. vaginalis foi de 1,02\%. Conclusão: Identificou-se baixa prevalência de tricomoníase no município, mas foram identificadas outras IST's de elevada importância epidemiológica. Além disso, a pesquisa traz uma inquietação sobre o tema e importante contribuição científica por ser a primeira investigação da doença no estado do Pará e a segunda da região Norte do país.

Palavras-chave: Epidemiologia, Tricomoníase, Coinfecção, Atenção Primária à Saúde.

\begin{abstract}
Objective: To determine the prevalence of trichomoniasis and associated co-infections in women treated at two health centers located in a municipality in the state of Pará and to correlate this information with sociodemographic variables. Methods: This is a retrospective, observational study, with a quantitative approach, carried out in two health units in the state of Pará, between March and December 2019. The sample consisted of 520 medical records of women attended between January 2012 to December 2017, aged between 18 and 55 years, showing a positive diagnosis for trichomoniasis and / or other infections. Results: The prevalence of $T$. vaginalis recorded for both units was $0.96 \%$. When associated with sociodemographic variables, they all resided in a municipality in the state of Pará and declared themselves brown; $0.58 \%$ were households, aged $28-32$ years, married and denied the use of contraceptives; only $0.38 \%$ had completed high school. The prevalence of co-infections involving T. vaginalis was $1.02 \%$. Conclusion: A low prevalence of trichomoniasis was identified in the municipality, but other STIs of high epidemiological importance were also identified. In addition, the research brings a concern about the theme and an important scientific contribution, as it is the first investigation of the disease in the state of Pará and the second in the northern region of the country.
\end{abstract}

Keywords: Epidemiology, Trichomoniasis, Coinfection, Primary Health Care.

1 Universidade do Estado do Pará (UEPA), Belém - PA. *E-mail: maizasouza1619@gmail.com.

2 Universidade da Amazônia (UNAMA), Belém - PA.

${ }^{3}$ Escola Superior da Amazônia (ESAMAZ), Belém - PA.

${ }^{4}$ Centro Universitário do Pará (CESUPA), Belém - PA. 


\section{RESUMEN}

Objetivo: Determinar la prevalencia de tricomoniasis y coinfecciones asociadas en mujeres atendidas en dos centros de salud ubicados en un municipio del estado de Pará y correlacionar esta información con variables sociodemográficas. Métodos: Se trata de un estudio observacional retrospectivo, con abordaje cuantitativo, realizado en dos unidades de salud del estado de Pará, entre marzo y diciembre de 2019. La muestra estuvo conformada por 520 historias clínicas de mujeres atendidas entre enero de 2012 y diciembre de 2017. edad entre 18 y 55 años, presentando diagnóstico de tricomoniasis y / u otras infecciones. Resultados: La prevalencia de $T$. vaginalis registrada para ambas unidades fue de $0,96 \%$. Al asociarse con variables sociodemográficas, todos vivían en el estado de Pará y se declaraban morenos; El 0,58\% eran del hogar, de 28 a 32 años, casados y se les negó el uso de anticonceptivos; sólo el 0,38\% había completado la escuela secundaria. La prevalencia de coinfecciones por $T$. vaginalis fue del 1,02\%. Conclusión: Se identificó una baja prevalencia de tricomoniasis en el municipio, pero se identificaron otras ITS de alta importancia epidemiológica. Además, la investigación trae consigo una preocupación por el tema y un importante aporte científico ya que es la primera investigación de la enfermedad en el estado de Pará y la segunda en la región norte del país.

Palabras clave: Epidemiología, Tricomoniasis, Coinfección, Atención Primaria de Salud.

\section{INTRODUÇÃO}

A tricomoníase é conhecida como a infecção sexualmente transmissível (IST) não viral mais recorrente, e que apresenta um elevado nível de incidência anualmente em todo o mundo. Dados da Organização Mundial da Saúde avaliou o número de 275,4 milhões de novos casos da doença em adultos com idades entre 15 e 49 anos, em 2008. No Brasil, um levantamento epidemiológico realizado pela Coordenação Nacional de DST do Ministério da Saúde, em 2001, identificou um total de 208.500 casos de homens e 2.672 .200 casos de mulheres infectados por Trichomonas vaginalis (ALVES MJ, et al., 2011; FILHO HMT e LEITE CCF, 2015; SOUZA VS, 2016).

Estudos apontam a prevalência da doença em alguns municípios e regiões, como o de Lima MCL, et al. (2013) que verificou a prevalência de 10,5\% no Nordeste do Brasil, em pesquisa realizada no município de Vitória de Santo Antão, e outra em São Paulo e na Alta Sorocabana, Dan VJL, et al. (2013) encontrou a prevalência de $0,63 \%$ e $0,54 \%$, respectivamente, de mulheres infectadas por $\mathrm{T}$. vaginalis. E no município de Porto Velho, Roraima, Souza VS (2016) encontrou 118 casos de mulheres infectadas, o que corresponde à prevalência de $0,57 \%$.

A tricomoníase é causada pelo patógeno $T$. vaginalis, protozoário flagelado que pertence à família Trichomonadidae, cujo primeiro relato data de 1836. Seu habitat é o trato geniturinário dos seres humanos, onde sua presença é responsável por várias complicações, como dispareunia, disúria e frequência miccional, nas mulheres; e prostatite, balanopostite e cistite, nos homens. Geralmente, trata-se de uma infecção assintomática nestes (NEVES DP, 2005; DIÉGUEZ IS, 2014; ZHON S, 2016).

E ainda, pesquisas revelam que $T$. vaginalis está fortemente associado ao desenvolvimento de câncer cervical e atua como facilitador de outras IST's. Devido à dilatação dos vasos sanguíneos e o surgimento de pontos hemorrágicos na mucosa, que é um sinal característico da tricomoníase, há o acesso direto para a corrente sanguínea. Sendo assim, há uma facilitação da transmissão do Vírus da Imunodeficiência Humana (HIV) de indivíduos HIV-positivos para o parceiro HIV-negativo, caso o primeiro apresente co-infecção ativa por T. vaginalis. Estima-se a incidência anual de 746 novos casos de HIV decorrentes da facilitação da tricomoníase (SOUZA VS, 2016; FICHOROVA RN, et al., 2012; LEMOS PAP e AMARAL WN, 2015; AMBROZIO CL, et al., 2016).

T. vaginalis apresenta elevada resistência, pois estudos já demonstraram que pode permanecer viável por três horas na urina e seis horas no sêmen ejaculado; em ambientes úmidos pode sobreviver até 24 horas á temperatura de $35^{\circ} \mathrm{C}$, enquanto nos locais secos é capaz de resistir por vários minutos em exposição à luz solar direta. Esse potencial de resistência favorece a capacidade de disseminação do parasito e também sua infectividade (SOUZA VS, 2016; DIÉGUEZ IS, 2014). Ainda, segundo Castro AA (2013) o parasito pode permanecer resistente por mais de uma semana sobre o prepúcio de um homem contaminado, e de acordo 
com Consolaro MEL e Maria-Engler SS (2012) ele sobrevive por mais de 48 horas no exsudato vaginal a 10 ㅇ $\mathrm{C}$ e por um período de 24 horas em toalhas de pano umedecidas a temperatura de $35{ }^{\circ} \mathrm{C}$. Esses dados revelam o potencial de infectividade de $T$. vaginalis e ratifica o risco de contaminação através do compartilhamento de objetos pessoais e a prática sexual desprotegida.

A transmissão deste protozoário ocorre principalmente através das relações sexuais desprotegidas, mas também, o uso de banheiros públicos, saunas, compartilhamento de toalhas de banho e outros fômites, material ginecológico não esterilizado, são relatados como outras formas de contaminação (SOUZA VS, 2016; MACHADO ER e SOUZA LP, 2012; LEMOS PAP e GARCÍA-ZAPATA MTA, 2014; WEBER AV e BACKERS $\mathrm{LTH}, 2016)$. Outro aspecto importante refere-se aos fatores de risco associados à tricomoníase, em que são apontadas as condições socioeconômicas, o nível de instrução escolar, sexo desprotegido e também a faixa etária, pois alguns estudos revelam maior prevalência da doença em mulheres com idades entre 45 e 50 anos (Lima MCL, et al., 2013; Dan VJL, et al., 2013; MACHADO ER e SOUZA LP, 2012; NETO PADM, et al., 2014).

Ressalta-se a relevância da presente pesquisa por ser o primeiro registro de informações epidemiológicas sobre a tricomoníase no Estado do Pará, podendo servir de subsídio para a realização de novos estudos e auxiliar na gestão dos serviços de saúde, assim como direcionar as ações voltadas à promoção, prevenção, diagnóstico e tratamento da tricomoníase, visando à melhoria dos serviços oferecidos a população. Dessa forma, tem-se como objetivos determinar a prevalência de tricomoníase e coinfecções associadas em mulheres atendidas em dois centros de saúde localizados em um município do estado do Pará e correlacionar essas informações com variáveis sociodemográficas das usuárias.

\section{MÉTODOS}

Realizou-se um estudo retrospectivo, observacional, de corte transversal, com abordagem quantitativa e analítica. Segundo Fontelles MJ (2012), os estudos de corte transversal, quando realizados em populações bem definidas, permitem obtenção de medidas de prevalência de fatores de risco e frequência de casos prevalentes de doença, sendo, por isso, chamados de estudos de prevalência. A pesquisa quantitativa trabalha com variáveis expressas sob a forma de dados numéricos, empregando recursos para classificá-las e analisá-las. Em razão de sua maior precisão e confiabilidade, os estudos quantitativos são mais indicados para o planejamento de ações coletivas.

A pesquisa foi realizada em duas unidades de saúde localizadas em um município do estado do Pará, com universo amostral composto por 520 prontuários de mulheres atendidas entre janeiro de 2012 a dezembro de 2017. Os critérios de inclusão foram prontuários de mulheres com idades entre 18 e 55 anos, apresentando diagnóstico positivo para tricomoníase e/ou outras infecções e a presença de informações sociodemográficas das participantes da pesquisa. Foram excluídos da pesquisa todos os prontuários cujas informações sobre 0 objeto da investigação não estavam presentes e aqueles de pacientes cuja faixa etária era inferior a 18 anos de idade.

Os dados foram coletados conforme a disponibilidade do local, no período de março a dezembro de 2019, com as visitas agendadas previamente com os profissionais que trabalhavam no arquivo e as informações registradas em um formulário próprio, contendo as variáveis: idade, raça/cor, procedência, naturalidade, escolaridade, profissão, estado civil, número de parceiros, uso de anticoncepcional e resultado de exames positivos para tricomoníase e/ou outras infecções. Os dados coletados foram armazenados em Planilhas Microsoft Excel 2010 e submetidos à análise de prevalência.

A pesquisa foi realizada após assinatura do Termo de Consentimento de Utilização dos dados pela coordenação dos Centros de Saúde, permitindo a realização da pesquisa com liberação dos prontuários para coleta de dados e a aprovação do Comitê de Ética em Pesquisa do Curso de Graduação em Enfermagem da Universidade do Estado do Pará sob o No CAAE 98422718.6.0000.5170 e Parecer de número: 2.945.752. Foi resguardada a identidade das pacientes por meio da utilização de código numeral (COD 01) para identificação dos formulários e não foram coletados quaisquer dados que pudessem identificar as usuárias, em consonância com a Resolução 466, de 12 de dezembro de 2012 do Conselho Nacional de Saúde (CNS), que normatiza a pesquisa com seres humanos. 


\section{RESULTADOS E DISCUSSÃO}

Os dados que compõe este estudo foram obtidos de 520 prontuários de pacientes do sexo feminino atendidas nas Unidades Municipais de Saúde de dois bairros populosos de um município do Estado do Pará, sendo identificadas como Unidade 1 (U1) e Unidade 2 (U2), correspondendo 62,12\% (323/520) e 37,88\% (197/520), respectivamente.

$\mathrm{Na}$ Unidade 1 (U1) identificou-se apenas 0,31\% (1/323) caso de paciente infectada com $T$. vaginalis. A Unidade 2 (U2), por sua vez, apresentou 3,05\% (6/197) de mulheres infectadas com este protozoário, e destes $1,02 \%(2 / 197)$ estavam associados à Candida sp. e G. vaginalis, respectivamente, correspondendo a 2 casos de coinfecção na U2 (Gráfico 1).

Gráfico 1 - Prevalência de T. vaginalis e coinfecções encontradas em duas unidades de saúde, (U1) e (U2), em um município do Pará, 2019.

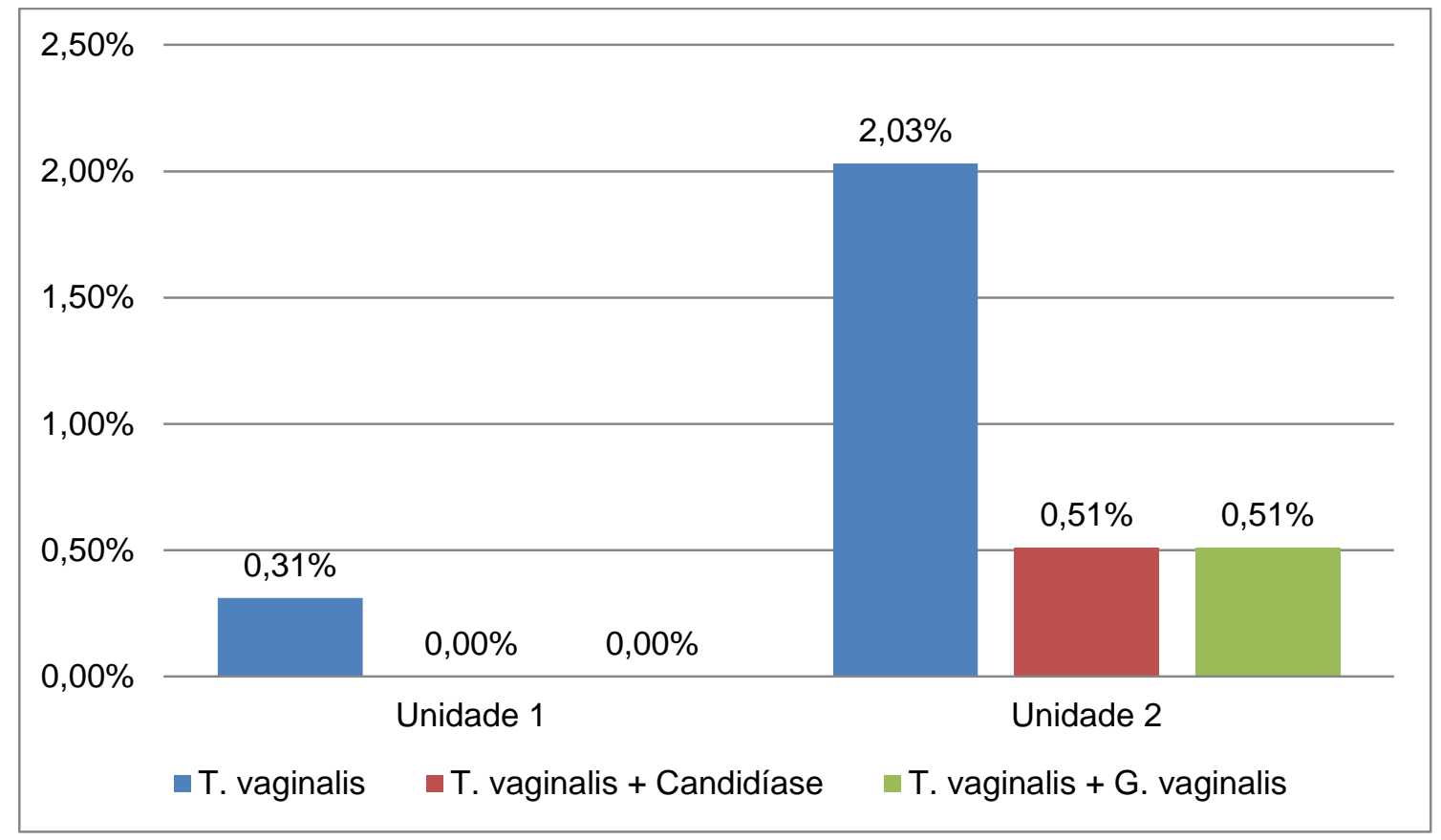

Legenda: a prevalência calculada baseia-se nos dados de cada unidade: U1: 323 (100\%); U2: $197(100 \%)$.

Fonte: Sousa MS, et al., 2020.

Considerando os dados das duas unidades, a prevalência de $T$. vaginalis registrada foi de $0,96 \%(5 / 520)$ casos. Quando associada a variáveis socioeconômicas, todas residiam no município estudado e se autodeclararam pardas; $0,58 \%$ (3/520) eram do lar, com idades entre 28-32 anos, casadas e informaram apenas um parceiro sexual, negando o uso de anticoncepcional; apenas $0,38 \%$ (2/520) possuíam ensino médio completo e as demais, o nível de escolaridade inferior a este.

Estudo realizado por Luppi CG, et al. (2011), obteve prevalência de $3,2 \%$ para $T$. vaginalis em 781 amostras de secreção vaginal de pacientes atendidas em um centro de atenção primária na região metropolitana de São Paulo. Em outro estudo realizado por Dan VJL, et al. (2013), em São Paulo e na Alta Sorocabana, usando dados do Sistema de Informações do Câncer do Colo do Útero (SISCOLO), a prevalência de mulheres infectadas por $T$. vaginalis foi de $0,63 \%$ e $0,54 \%$, respectivamente. Esses resultados são semelhantes aos encontrados em nossa pesquisa, evidenciando uma distribuição similar da prevalência da tricomoníase em estados com índices de desenvolvimento diferentes.

Da mesma forma, estudo realizado em Goiás por Camargo KC, et al. (2015), com 302 mulheres, revelou positividade para este protozoário em $2 \%$ das pacientes. Na região Norte, em estudo desenvolvido por Souza VS (2016), no município de Porto Velho e Roraima, utilizando 20.701 dados obtidos no Sistema de 
Informações do Câncer do Colo do Útero (SISCOLO) no período de janeiro de 2014 a fevereiro de 2015 mostraram $118(0,57 \%)$ pacientes infectadas pelo $T$. vaginalis. Os resultados destes estudos corroboram com os obtidos na presente pesquisa.

A prevalência em outras cidades e regiões brasileiras, como no Nordeste, Centro-oeste e Sul, apresentam algumas variações, como mostra o estudo transversal realizado no Nordeste por Lima MCL, et al. (2013), utilizando dados de 314 prontuários, o qual verificou a prevalência de $T$. vaginalis em 10,5\% das mulheres, associando a presença de hipertensão arterial, odor vaginal desagradável e dispareunia como fatores de risco independentes da infecção. Assim como, pesquisa realizada por Ambrozio CL, et al. (2016) em 19 municípios do Rio Grande do Sul utilizando o método de análise a fresco em 300 mulheres, identificou positividade em $9 \%$ das pacientes. Esses resultados diferem dos encontrados nas pesquisas anteriormente relatadas e que corroboram o nosso estudo.

Tal discrepância nos resultados pode estar associado ao método empregado no diagnóstico de $T$. vaginalis, pois de acordo com Filho HMT e Leite CCF (2015) o padrão ouro utilizado na identificação do protozoário é a cultura, em que são utilizados meios específicos. Este método, porém, apresenta algumas limitações, como demanda por maior tempo de mão de obra especializada e o diagnóstico é mais demorado, além de não ser encontrado amplamente nos laboratórios. Dessa forma, os métodos mais utilizados na rotina são a pesquisa a "fresco" e a coloração de Papanicolau, que apresentam sensibilidade bastante variável, pois dependem da experiência do observador. (Filho HMT e Leite CCF, 2015).

Essa pode ser uma justificativa para a variação acentuada nos valores da prevalência de $T$. vaginalis registrada nos estudos já apresentados. Assim como, para a baixa prevalência encontrada nas duas unidades de saúde da presente pesquisa, uma vez que os métodos diagnósticos registrados nos prontuários das pacientes foram o citológico de Papanicolau e a secreção vaginal (não foi especificado se era o método a fresco). Uma vez que o diagnóstico preciso de $T$. vaginalis utilizando essas metodologias requer a presença do protozoário em grande quantidade (citologia oncótica de Papanicolau) e depende da experiência do observador, podemos sugerir que há um subdiagnóstico da infecção por $T$. vaginalis.

Em relação as coinfecções, a prevalência registrada foi de 1,02\% (2/197), com registro apenas na U2. Os dois casos de coinfecções identificadas são de T. vaginalis + Candida sp. e T. vaginalis + Gardnerella vaginalis, correspondendo a uma prevalência de $0,51 \%$, respectivamente. A literatura revela uma associação frequente entre T. vaginalis + $G$. vaginalis, como pode ser observado no estudo de Martins RA, et al. (2018) que registrou prevalência de 3,19\% para essa coinfecção.

Enquanto a associação entre $T$. vaginalis + Candida sp. é menos comum, com uma prevalência de 0,05\% registrada em estudo observacional, retrospectivo, que analisou 2.191 resultados de exames colpocitológicos em uma unidade de saúde do município de Vista Serrana, Paraíba. (MARTINS RA, et al., 2018).

Em estudo realizado por Teixeira PM (2018), com amostra de 341 mulheres, foram realizados alguns testes diagnósticos como bacterioscopia pós-coloração de Gram, método citológico e a reação em cadeia da polimerase (PCR), com a finalidade de identificar os patógenos presentes na amostra. Do total de participantes, foi selecionado 80 amostras para a realização da PCR afim de detectar T. vaginalis, Neisseria gonorrhoeae, Chlamydia trachomatis e Papiloma Vírus Humano (HPV). Foi identificada a presença de coinfecção em $15 \%$ da amostra e destas, $25 \%$ era de Chlamydia trachomatis $+T$. vaginalis e $16,7 \%$ era de $T$. vaginalis + HPV. Esse resultado mostra que além da associação com patógenos mais comuns, identificada em nossa pesquisa, $T$. vaginalis, pode apresentar coinfecção com outros patógenos.

Quanto as variáveis socioeconômicas, 1,02\% dos casos de coinfecção tinham ensino médio completo, eram do lar, casadas e informaram apenas um parceiro sexual; $T$. vaginalis $+G$. vaginalis esteve presente em quem fez uso de anticoncepcional, com idade entre 23-27 anos e autodeclarada branca. Enquanto que a associação de $T$. vaginalis + Candida $s p$. esteve presente em quem não fazia uso de anticoncepcional, com intervalo de idade entre 18-22 anos e autodeclarada parda.

Além da tricomoníase e suas coinfecções, foi identificado em nosso estudo a presença de outros microrganismos de forma isolada, como G. vaginalis, Candida sp., Treponema pallidum, Chlamydia 
trachomatis, Citomegalovírus, Vírus da Imunodeficiência Humana (HIV), Papiloma Vírus Humano (HPV), Vírus T-Linfotrópico Humano (HTLV-1 e HTLV-2), bem como vários casos de coinfecções entre eles. Dentre os quais se destacam, pela frequência ou pela gravidade da infecção: $G$. vaginalis + Candida sp., G. vaginalis + Treponema pallidum, G. vaginalis + HPV, HIV + HPV, Treponema pallidum + Citomegalovírus, HTLV 1 + HTLV 2, e ainda houve um caso de tripla infecção com G. vaginalis + Candida sp. + Treponema pallidum (Gráfico 2).

Gráfico 2 - Prevalência das infecções isoladas encontradas em duas unidades de saúde, (U1) e (U2), em um município do Pará, 2019.

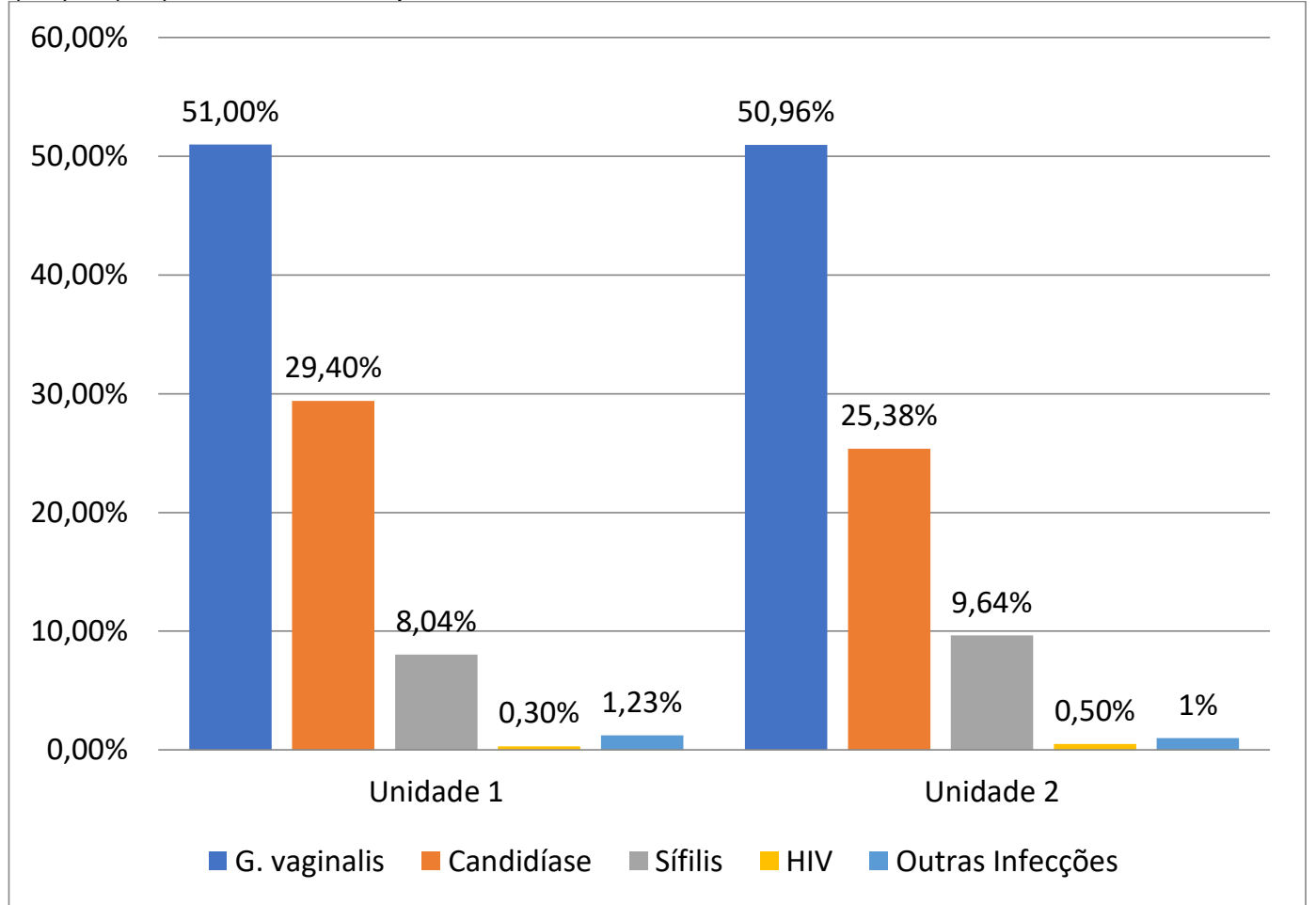

Legenda: a prevalência calculada baseia-se nos dados de cada unidade: U1: 323 (100\%); U2: $197(100 \%)$.

Fonte: Sousa MS, et al., 2020.

Ao calcular a porcentagem absoluta dos dados obtidos nas duas unidades pesquisadas obtêm-se, 50,96\% (265/520) para Gardnerella vaginalis, 27,88\% (145/520) para Candidíase, 8,65\% para Sífilis (45/520), 0,38\% (2/520) para HIV e 1,15\% (6/520) para ouras infecções. Em outras infecções estão presentes microrganismos como: HTLV, tipo 1 e 2; HPV e citomegalovírus.

Alguns resultados obtidos são corroborados pelo estudo de Dall'alba MP e Jaskulski MR (2014), no qual foram analisados 293 prontuários de mulheres entre os anos de 2007 e 2009, obtendo prevalência de $51 \%$ para G. vaginalis, $15 \%$ para candidíase (Candida sp.) e 3\% para outras infecções. Outro estudo realizado por Muniz SDB, et al. (2019) em mulheres com idade entre 18 a 30 anos em uma unidade básica de saúde do município de Cajazeiras-PB, encontrou prevalência de 29,68\% para candidíase. Esses resultados se aproximam dos encontrados em nossa pesquisa, evidenciando que essas infecções são bastante frequentes no público alvo investigado.

Ainda, em pesquisa realizada por Silva DAR, et al. (2017) na qual foram avaliados 1300 prontuários, entre 2012 e 2014 em um serviço de referência de Porto Alegre, registrou-se 7,9\% de mulheres infectadas por sífilis (Treponema pallidum). O Boletim Epidemiológico emitido pelo Ministério da Saúde em 2012 revela 0,6\% de prevalência de HIV em pacientes com idades entre 15 a 49 anos. Os dados encontrados nesses estudos vão de encontro aos resultados obtidos na presente pesquisa, o que nos permite sugerir que a distribuição dessas infecções no estado do Pará apresenta uma prevalência semelhante as demais cidades brasileiras avaliadas. (BRASIL, 2012). 
Ao calcular a porcentagem absoluta das coinfecções para as duas unidades, o resultado foi $4,42 \%$ de coinfecção G. vaginalis + candidíase; $0,96 \%$ candidíase + sífilis; $2,30 \%$ G. vaginalis + sífilis; $0,38 \%$ HPV + candidíase e $1,53 \%$ em outras coinfecções. Neste último grupo estão incluídas coinfecções de G. vaginalis + citomegalovírus, HIV + HPV, G. vaginalis + Chlamydia trachomatis, sífilis + citomegalovírus e um caso de tripla infecção de G. vaginalis + candidíase + sífilis, (Gráfico 3).

Gráfico 3 - Principais coinfecções encontradas em duas unidades de saúde, (U1) e (U2), em um município do Pará, 2019.

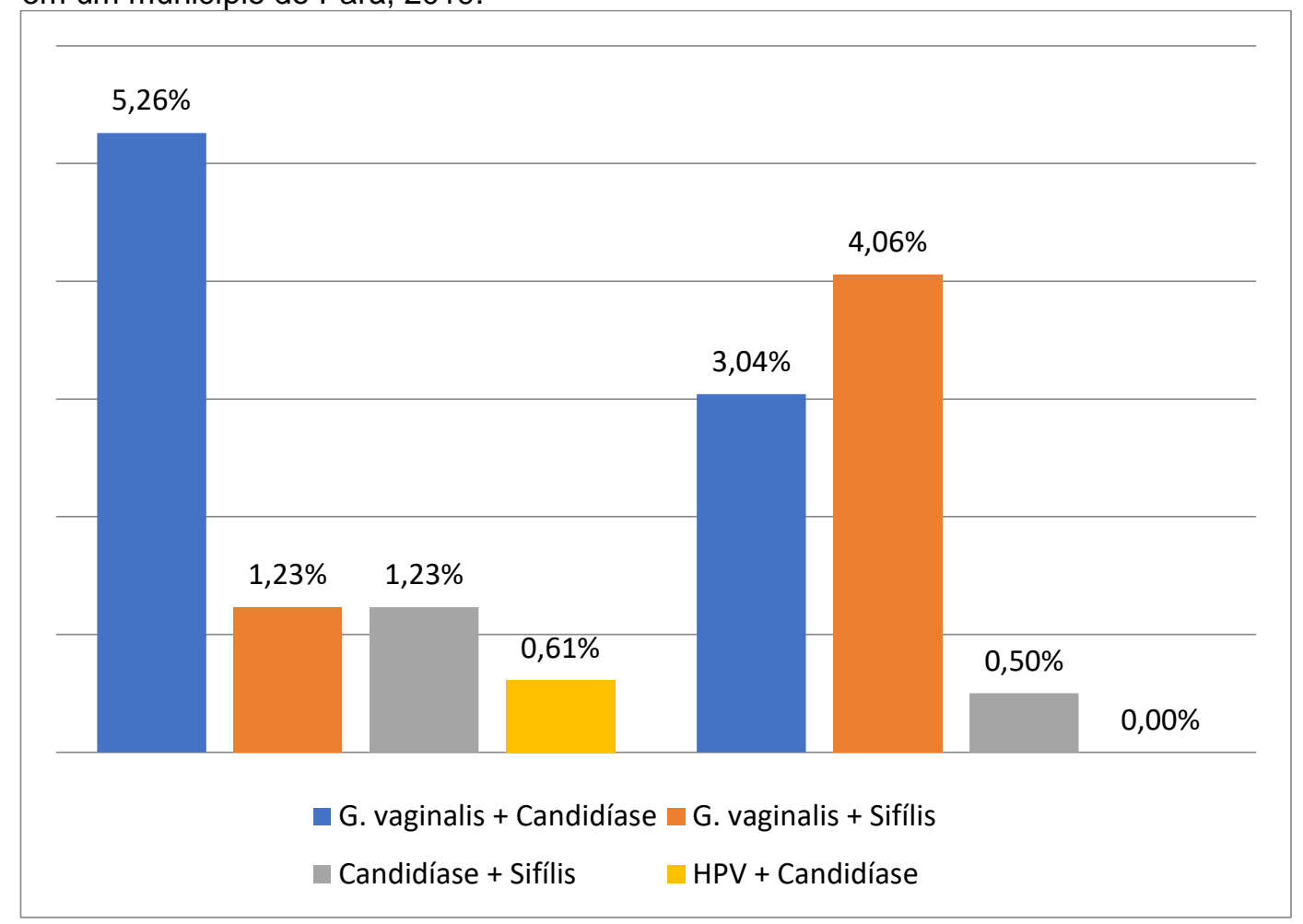

Legenda: a prevalência calculada baseia-se nos dados de cada unidade: U1: 323 (100\%); U2: $197(100 \%)$.

Fonte: Sousa MS, et al., 2020.

Em relação às variáveis sociodemográficas, na U1 34,67\% apresentaram idade entre 23 e 27 anos, $88,85 \%$ eram pardas, $92,26 \%$ residiam no município estudado, $52,63 \%$ eram do lar e $36,53 \%$ tinham o ensino médio completo. Três das variáveis oferecem uma avalição geral do comportamento sexual das mulheres estudadas, sendo identificadas como: o estado civil, em que 78,94\% da amostra eram casadas, o número de parceiros, em que $65,01 \%$ apresentaram apenas um parceiro sexual e o uso de anticoncepcional, onde $53,56 \%$ informaram não fazer uso desse método contraceptivo.

$\mathrm{Na}$ U2 as variáveis indicaram que $22,8 \%$ das mulheres estavam entre a faixa etária de 18 a 22 anos, $72 \%$ declararam-se pardas, $81,2 \%$ eram residentes do município estudado, tendo também $30,4 \%$ de mulheres do lar e $51,7 \%$ com ensino médio completo. Já para os dados obtidos sobre a avaliação geral do comportamento sexual das mulheres presentes na pesquisa, obteve-se, em relação ao estado civil, que $71 \%$ delas são casadas, $83,2 \%$ já tiveram entre 1 a 3 parceiros e $57,8 \%$ relataram fazer uso de anticoncepcional.

Estudo realizado por Teixeira PM (2018), no município de Ouro Preto, Minas Gerais, para investigar os fatores associados a prevalência de vaginoses bacterianas, identificou que $50,1 \%$ das mulheres apresentavam idade superior a 40 anos, 53,4\% eram casadas ou tinham parceiro fixo, $48,7 \%$ tinham ensino médio completo ou incompleto, 39,4\% se autodeclararam pardas ou de origem asiático-indígena e 51,6\% não faziam uso de anticoncepcional oral. Esses dados se mostram bastante variados quando comparados aos resultados do nosso estudo, o que sugere a diversificação de fatores sociais e epidemiológicos relacionados a presença de vaginoses bacterianas, assim como de outras infecções em mulheres. 
Assim, a partir desses resultados, pode-se observar que houve uma grande variedade de infecções na população estudada, distribuídas em frequências e graus de risco variados, desde uma vaginose bacteriana resultante de desequilíbrio de flora vaginal (Candidíase e Gardnerelose) até casos de infecção por HIV, HPV e HTLV, que requerem um manejo mais complexo.

\section{CONCLUSÃO}

Identificou-se baixa prevalência de tricomoníase no município estudado, todavia, esse resultado é questionado, pois acredita-se em um possível subdiagnóstico de $T$. vaginalis, uma vez que os métodos diagnósticos empregados na identificação do protozoário apresentam algumas limitações e os sinais e sintomas clínicos descritos nos prontuários sugerem a presença do parasita em muitas usuárias. Dessa forma, ressalta-se a importância da investigação clínica e laboratorial criteriosa para que os casos não sejam subdiagnosticados, e consequentemente, fique sem tratamento, já que a doença provoca muito desconforto na mulher, além de estar associada a complicações, como câncer cervical, facilitar a infecção por HIV e outros patógenos. Assim, acredita-se que a presente pesquisa foi de grande relevância ao preencher uma lacuna sobre os dados relativos à infecção por $T$. vaginalis no estado do Pará, complementando os dados da literatura referente as informações epidemiológicas da Região Norte do Brasil.

\section{REFERÊNCIAS}

1. ALVES MJ, et al. Epidemiologia de Trichomonas vaginalis em mulheres. Rev Port Saude Publica. 2011; 29(1): 27-34p.

2. AMBROZIO CL, et al. Trichomonas vaginalis prevalence and risk factors for women in Southern Brazil. Rev Inst Med Trop Sao Paulo. 2016; 58(61): 1-5p.

3. BRASIL. MINISTERIO DA SAÚDE. Boletim Epidemiológico. AIDS no Brasil: epidemia concentrada e estabilizada em populações de maior vulnerabilidade. Brasília. 2012; 43(1).

4. CAMARGO KC, et al. Secreção vaginal anormal: sensibilidade, especificidade e concordância entre o diagnóstico clínico e o citológico. Rev Bras Ginec Obstet. 2015; 37(5): 222-228p.

5. CASTRO AA. Atuação do enfermeiro frente a pacientes com tricomoníase: revisão integrativa da literatura. Monografia (Graduação em Enfermagem) - Universidade de Brasília, Faculdade de Ceilândia, Distrito Federal, 2013.

6. CONSOLARO MEL, MARIA-ENGLER SS. Citologia clínica cervicovaginal. São Paulo: Roca, 2012.

7. DALL'ALBA MP, JASKULSKI MR. Prevalência de vaginoses bacterianas causadas por Gardnerella vaginalis, em um laboratório de análises clínicas na cidade de santo expedito do Sul, RS. Perspectiva, Erechim [periódico na Internet]. 2014 Mar [acessado 2020 Fev 25]; 38: [cerca de 9 p.]. Disponível em: http://www.uricer.edu.br/site/pdfs/perspectiva/1002_412.pdf.

8. DAN VJL, et al. Prevalência de tricomoníase na Alta Sorocabana e no Estado de São Paulo. Colloquium Vitae. 2013; 5(1): 30-39p.

9. DIÉGUEZ IS. Tricomoníasis: uma visión amplia. Rev Med Universid Antioquia - IATREIA. 2014; 27(2): 198-205p.

10. FICHOROVA RN, et al. Endobiont viruses sensed by the human host-beyond conventional antiparasitic therapy. PLOS ONE. 2012; 7(11): 1-16p.

11. FILHO HMT; LEITE CCF. Doenças sexualmente transmissíveis curáveis: Diagnóstico laboratorial. Jornal Bras Med. 2015; 103(1): 17-24p.

12. FONTELLES MJ. Bioestatística aplicada à pesquisa experimental. 1. ed. São Paulo: Editora Livraria da Física, 2012.

13. LEMOS PAP, AMARAL WN. Trichomonas vaginalis e sua associação com o câncer cervical: uma revisão sistemática. FEMINA: Rev Fed Bra Assoc Ginec Obstet. 2015; 43 (5): 209-214p.

14. LEMOS PAP, GARCÍA-ZAPATA MTA. Aspectos relacionados com a infecção por Trichomonas vaginalis e diagnóstico laboratorial. Acta Obstet Ginec Portug. 2014; 8(2): 152-162p.

15. LIMA MCL, et al. Prevalência e fatores de risco independentes à tricomoníase em mulheres assistidas na atenção básica. Acta Paul Enferm. 2013; 26(4): 331-337p.

16. LUPPI CG, et al. Diagnóstico precoce e os fatores associados às infecções sexualmente transmissíveis em mulheres atendidas na atenção primária. Rev Bras Epidemiol. 2011; 14(3): 467-477p.

17. MACHADO ER, SOUZA LP. TRICOMONÍASE: Assistência de enfermagem na prevenção e controle. Ensaios Ciencia: Ciencias Biol Agrarias Saude. 2012; 16(4): 229-243p.

18. MARTINS RA, et al. Frequência de Trichomonas vaginalis, Gardnerella vaginalis e Candida spp. Em exames colpocitológicos em Vista Serrana-PB. Rev Cienc Saude Nova Esperança. 2018; 16(2): 28-37p.

19. MUNIZ SDB, et al. Prevalência de candidiase vulvovaginal recorrente em mulheres com idade entre 18 a 30 anos em uma unidade básica de saúde no município de cajazeiras - PB. Journal of Biology \& Pharmacy and Agricultural Management [periódico na Internet].

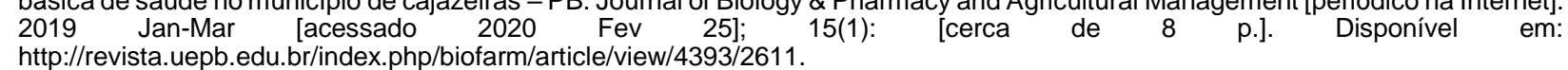

20. NETO PADM, et al. Inquérito comportamental sobre fatores de risco a Trichomonas vaginalis. UNOPAR Cient Ciencias Biol Saude. $2014 ; 16(1): 9-13 p$.

21. NEVES DP. Parasitologia Humana. 11. ed. São Paulo: Atheneu; 2005.

22. SILVA DAR, et al. Prevalência de sífilis em mulheres. Enferm Foco [periódico na Internet]. 2017 [acessado 2020 Fev 25]; 8(3): [cerca de 8 p.]. Disponível em: http://revista.cofen.gov.br/index.php/enfermagem/article/view/891/401.

23. SOUZA VS. Prevalência de Trichomonas vaginalis em mulheres atendidas no sistema único de saúde (SUS) no município de Porto Velho/Ro nos períodos de 2014-2015. Monografia (Graduação em Biomedicina) - Centro de Ensino São Lucas. Porto Velho, Roraima, 2016.

24. TEIXEIRA PM. Prevalência e fatores associados à vaginose bacteriana em mulheres atendidas pelo SUS no município de Ouro Preto/MG. Dissertação (Mestrado em Ciências Farmacêuticas) - Escola de Farmácia, Universidade Federal de Ouro Preto, Minas Gerais, 2018; 83p.

25. WEBER AV, BACKERS LTH. Análise retrospectiva de inflamações cervicovaginais causadas por agentes microbiológicos no sul do Brasil. Rev Saude Integ. 2016; 9(17): 28-40p.

26. ZHON S. Tricomoniasis. Monografia (Grado en Farmacia) - Departamento de Microbiología y Parasitología. Universidad de Sevilla. Sevilla, 2016. 